\section{National Poster Prize Winners}

\section{NP-001 PATIENTS WITH MULTIPLE SCLEROSIS TREATED WITH HIGH DOSES OF BIOTIN: PREVENTION OF SIGNIFICANT BIOLOGICAL EXAMINATION DISTURBANCES BY HOSPITAL PHARMACISTS}

${ }^{1} \mathrm{~A}$ Bacle, ${ }^{2} \mathrm{~B}$ Hue, ${ }^{2} \mathrm{C}$ Lebert, ${ }^{2} \mathrm{~B}$ Troussier, ${ }^{1} \mathrm{P}$ Le Corre, ${ }^{2} \mathrm{~V}$ Gicquel. ${ }^{1}$ Service de Pharmacie et Laboratoire de Biopharmacie et Pharmacie Clinique, Hôpital Pontchaillou, CHU de Rennes et Faculté de Pharmacie, Rennes; ${ }^{2}$ Service Pharmacie, Hôpital Pontchaillou, Rennes

\subsection{6/ejhpharm-2019-eahpconf.626}

Background Biotin at high dose $(300 \mathrm{mg} / \mathrm{d})$ is used in primary or secondary forms of multiple sclerosis (MS) (temporary use authorisation in France). Some biological tests are biased by this treatment, including immunoassays using biotin as a reagent. The results are likely to be overestimated for competitive assays (eg thyroid hormones) and underestimated for immunometric assays (eg thyroid-stimulating hormone).

In 2007, the Agence Nationale de Sécurité du Médicamentdrew the attention of biologists and hospital directors.

Pharmacists, despite their primary role in patient care, have been forgotten.

Purpose This work presents the organisation established by the hospital pharmacy in collaboration with neurologists, biologists and the patients.

Materials and methods Three types of actions have been established:

- Neurologists report to the pharmacist the initiation of treatment by biotin at high dose.

- Pharmacists update a shared file of patients with biologists.

- Biologists integrate this information into validation software in order to neutralise biotin in the serum before assaying.

Outpatients are informed during the drug dispensation and receive a card entitled 'patient treated with high dose biotin' to present to all health professionals. All pharmacist who can deliver biotin have been trained.

Results A series of 52 patients were monitored in our establishment on 1 October 2018.

This initiative enabled us to sensitise the different stakeholders to this problem: retrospectively, disturbed thyroid hormone dosages results were found. The literature also cites false normal values of troponinaemia in the context of myocardial infarction and low-dose disturbances $(15 \mathrm{mg} / \mathrm{d})$. This collaborative work must enable the avoidance of this type of error.

Conclusions The central role of hospital pharmacists at the interface between patients, prescribers and biologists has been essential in establishing a strategy to limit biological interference. The verbal exchange between the patient and medical team remains one of the best means of prevention. The regional association of MS patients has also been contacted to relay this entire device to other facilities. General practitioners and pharmacists should also be warned. This information relay reinforces the importance of reasoning on the entire ambulatory-hospital pathway, especially since an extension of the indications is envisaged.

\section{REFERENCES AND/OR ACKNOWLEDGEMENTS}

None

\section{NP-002 MEDICATION RECONCILIATION AND MEDICATION REVIEW IN THE UROLOGICAL-ONCOLOGICAL OUTPATIENT CLINIC}

A Kähkönen, L Schepel, H Tolonen, M Utriainen, T Utriainen, M Airaksinen.

\subsection{6/ejhpharm-2019-eahpconf.627}

Background Internationally, clinical pharmacy services in oncology are usually patient-oriented and often include medication reconciliations and reviews. There is a need to find out how clinical pharmacists can improve medication safety in the division of solid tumours of Helsinki University Central Cancer Centre.

Purpose The aim of this study was to find out the accuracy of the medication charts and identify drug-related problems (DRPs) among over 65-year-old patients using six or more medicines in the urological-oncological outpatient clinic.

Materials and methods When the patient arrived at the urology-oncology outpatient clinic, the accuracy of the medication charts was assessed by pharmacist-led medication reconciliation, including patient interview. Information concerning patient's medication was also searched for from the national electronical prescription centre and from the records of previous hospital visits. DRPs, such as drug-drug interactions, adverse drug reactions and overlapping medications, were identified with the pharmacist-led medication review. Pharmacists discussed the clinical relevance of DRPs with the oncology specialist.

Results Altogether, 100 patients with urological cancer were included in this study. On average, they were 73 years' old and used 12 medications. On average, there were six discrepancies per patient in the hospital medication chart. Only two patients had a correct medication chart. The discrepancies were most commonly related to paracetamol $(n=38)$, pantoprazole $(n=29)$ and metoclopramide $(n=19)$. The most common discrepancies of high-alert medications were related to oxycodone $(n=17)$, the combination of paracetamol and codeine $(n=10)$, and enoxaparin $(n=10)$. In the medication review process, 139 DRPs were identified with 70 patients (two per patient). Of these DRPs, 70\% were regarded as clinically relevant and lead to actions by the oncology specialist. Reconsidering the need or efficacy of the medication (39\%) or medication adjustment due to renal insufficiency (17\%) were most commonly identified with medication reviews. DRPs were usually related to non-oncological medications such as pantoprazole $(n=19)$, the combination of calcium and vitamin-D $(n=9)$, and codeine $(n=7)$.

Conclusions The medication reconciliation process should be developed in the urology-oncology outpatient clinic. Multiprofessional medication reviews can be used to detect and resolve DRPs of older patients with urological cancer. The results of this study can be exploited when clinical pharmacy services are created and developed in the University Central Cancer Centre.

\section{REFERENCES AND/OR ACKNOWLEDGEMENTS}

Mekonnen AB, McLachlan AJ, Brien J-A . Effectiveness of pharmacist-led medication reconciliation programmes on clinical outcomes at hospital transitions: a systematic review and meta-analysis. BMJ Open 2016;6:

Schepel L, Lehtonen L, Airaksinen M, et al. Medication reconciliation and review for older emergency patients requires improvement in Finland. Int J Risk Safe Med 2019;30:19-31. 